\section{AB0228 CHILDREN WITH EXTENDED OLIGOARTICULAR AND POLYARTICULAR JUVENILE IDIOPATHIC ARTHRITIS HAVE A CYTOKINE PATTERN FAVOURING B CELL ACTIVATION IN CIRCULATION SIMILARLY TO EARLY AND ESTABLISHED RHEUMATOID ARTHRITIS PATIENTS}

Rita A. Moura ${ }^{1}$, Filipa Oliveira-Ramos ${ }^{1,2}$, Cláudia Marques ${ }^{1}$, Alexandre Brito ${ }^{1}$, Rui L. Teixeira ${ }^{1,2}$, Vasco Romão ${ }^{1,2}$, Raquel Campanilho-Marques ${ }^{1,2}$, Vítor Teixeira $^{2}$, Maria João Saavedra ${ }^{2}$, Cristina Ponte ${ }^{1,2}$, Nikita Khmelinskii ${ }^{2}$, Joao Eurico Fonseca'1,2. ${ }^{1}$ Unidade de Investigação em Reumatologia, Instituto de Medicina Molecular João Lobo Antunes, Faculdade de Medicina, Universidade de Lisboa, Lisbon, Portugal; ${ }^{2}$ Serviço de Reumatologia e Doenças Ósseas Metabólicas, Hospital de Santa Maria, CHULN, Lisbon Academic Medical Centre, Lisbon, Portugal

Background: The majority of polyarticular JIA (pJIA) and a large fraction of extended oligoarticular JIA (OJIA) patients fulfil classification criteria for rheumatoid arthritis (RA) in adulthood. B cells play several important roles in RA pathogenesis, but it is still unclear if the pattern of B cell involvement in PJIA and extended OJIA follows what has been described for adults with RA.

Objectives: The main goal of this study was to determine the concentration of cytokines potentially relevant for $B$ cell activation in serum from children with PJIA and extended OJIA when compared to children with persistent oJIA, adult JIA, early and established RA patients.

Methods: Serum samples were collected from children with extended oJIA $(n=8)$, persistent OJIA $(n=6)$, pJIA $(n=6)$, adult JIA $(n=8)$, untreated early RA $(<1$ year of disease duration, $n=12)$, established RA patients treated with synthetic DMARDs $(n=10)$ and two corresponding groups of age- and sex-matched healthy donors (children, $n=6$ and adults, $n=10$ ). A proliferation-inducing ligand (APRIL), B-cell activating factor (BAFF), interleukin (IL)- 6 and IL-21 serum levels were measured by ELISA.

Results: Children with extended oJIA, early and established RA patients had significantly higher BAFF serum levels when compared to controls, but no significant differences were observed in children with persistent OJIA, pJIA and adult JIA when compared to all groups included. APRIL serum levels were significantly increased in all patient groups when compared to controls, except in adult JIA, who had similar APRIL concentrations in comparison to controls. In addition, children with extended oJIA and PJIA had significantly higher APRIL serum levels when compared to adult JIA. IL-6 serum levels were significantly increased in children with extended oJIA, pJIA, early and established RA when compared to controls, but no significant differences were found in children with persistent oJIA and adult JIA patients. IL-21 serum levels were significantly increased in early RA when compared to controls, but no significant differences were observed between any of the other groups included.

Conclusion: The similarity in B cell cytokine pattern found between extended OJIA, pJIA, early and established RA patients, contrarily to what was observed in persistent oJIA, suggests an early $B$ cell involvement in the pathogenesis of extended oJIA and pJIA as described for RA.

Disclosure of Interests: Rita A. Moura: None declared, Filipa OliveiraRamos: None declared, Cláudia Marques: None declared, Alexandre Brito: None declared, Rui L. Teixeira: None declared, Vasco Romão: None declared, Raquel Campanilho-Marques: None declared, Vítor Teixeira: None declared, Maria João Saavedra: None declared, Cristina Ponte Speakers bureau: Roche, Nikita Khmelinskii: None declared, Joao Eurico Fonseca: None declared

DOI: 10.1136/annrheumdis-2019-eular.7201

\section{AB0229 RELATIVE MONOCYTE SUBSET DIFFERENCES BETWEEN JUVENILE- AND ADULT-ONSET SYSTEMIC LUPUS ERYTHEMATOSUS}

Hannah Peckham ${ }^{1}$, Lucia Martin-Gutierrez ${ }^{1,2}$, George Robinson $^{1,2}$, Anna Radziszewska ${ }^{1}$, Giulia Varnier ${ }^{2},{ }^{1,3}$ Claire Deakin $^{4}$, Nicolyn Thompson ${ }^{2}$ Elizabeth Jury ${ }^{2}$, Coziana Ciurtin ${ }^{1,2}$. ${ }^{1}$ Arthritis Research UK Centre for Adolescent Rheumatology, University College London, London, United Kingdom;

${ }^{2}$ Rheumatology Department, University College London, London, United Kingdom; ${ }^{3}$ Institute of Child Health, Great Ormond Street Hospital, London, United Kingdom; ${ }^{4}$ NIHR Biomedical Research Centre, Great Ormond Street Hospital, London, United Kingdom

Background: $15-20 \%$ of patients with Systemic Lupus Erythematosus (SLE) develop the disease in childhood or adolescence (Juvenile-onset SLE, JSLE) ${ }^{1}$. Whilst it is recognised that JSLE is often more severe than adult-onset $\mathrm{SLE}^{2}$, there is a lack of knowledge related to differences in the disease pathogenesis. Peripheral blood $\mathrm{CD}_{14}{ }^{+}$monocytes are thought to play a role in lupus development. $\mathrm{CD}_{16}{ }^{+}$Non-classical and
Intermediate monocyte subsets, producing inflammatory cytokines and contributing to T-cell activation and B-cell proliferation, are enriched in adults with $\mathrm{SLE}^{3}$. Furthermore, CD16 Classical monocytes are important for phagocytosis of apoptotic cells - a process known to be aberrant in lupus- contributing to the generation of autoantibodies. Thus we hypothesised that monocyte subsets could be differentially dysregulated in SLE and JSLE and relate to the disparities seen in pathogenesis and clinical presentation.

Objectives: To compare relative percentages of classical, intermediate and non-classical monocyte subsets in patients with SLE and JSLE.

Methods: Peripheral blood from female patients with SLE $(n=17)$ and JSLE $(n=23)$ was collected at the UCLH rheumatology clinics after obtaining informed consent. In depth phenotyping of peripheral blood mononuclear cells was performed using multiparameter flow cytometry. GraphPad Prism was used for Mann-Whitney U-tests and Spearman's Correlations, and ' $R$ ' for logistic regressions.

Results: Non-Classical CD14 dim $\mathrm{CD}_{16}{ }^{++} \quad(\mathrm{p}=0.007)$ and Intermediate $\mathrm{CD} 14_{\text {high }} \mathrm{CD}^{-} 6^{+}(\mathrm{p}=0.039)$ monocytes had significantly increased frequencies in SLE patients compared to those with JSLE while no significant differences were seen in Classical $\mathrm{CD} 14_{\text {high }} \mathrm{CD} 16^{-}$monocyte percentages between groups. Interestingly, non-classical monocytes correlated positively with age $(\mathrm{p}=0.006$, $r \mathrm{ho}=0.431)$, while classical monocytes negatively correlated with age $(p=0.042, \quad r h o=-0.323)$, suggesting a relationship between age and increasing proinflammatory monocyte phenotype. No relationship was observed between dsDNA titre or C3 levels and monocyte subset frequencies in SLE or JSLE patients. Classical monocyte frequencies negatively correlated with $E S R(p=0.027, r h o=-0.323)$ in JSLE patients, but this correlation was lost when JSLE and SLE were grouped together, or when SLE was examined in isolation. Increased percentages of non-classical monocytes were associated with a higher odds of adult onset SLE relative to JSLE, after adjusting for the effects of disease activity (SLE$\mathrm{DAl})(\mathrm{OR}=1.137,95 \% \mathrm{Cl}=1.021-1.266, \mathrm{p}=0.018)$.

Conclusion: Pro-inflammatory $\mathrm{CD}_{16}{ }^{+}$subset percentages were higher in adults and increased with age. Studies have shown a similar correlation in healthy adults ${ }^{4}$, suggesting that age-related differences in baseline immune cells may underpin differing mechanisms of monocyte involvement in lupus pathogenesis.

\section{REFERENCES :}

[1] Jimenez S. et al. (2003) The epidemiology of systemic lupus erythematosus. Clin Rev Allergy Immunology; 25: 3-12.

[2] Watson L. et al(2012) Juvenile-onset SLE; disease activity, severity and damage - the UK JSLE Cohort Study. Arthritis Rheum; 64: 2356-2365

[3] Zhu, H. et al(2016). CD16+ Monocyte subset was enriched and functionally exacerbated in driving T-cell activation and B-cell response in systemic lupus erythematosus. Frontiers in immunology; 7, 512.

[4] Seidler, S. et al(2010). Age-dependent alterations of monocyte subsets and monocyte-related chemokine pathways in healthy adults. BMC immunology; 11(1), 30.

Disclosure of Interests: None declared

DOI: 10.1136/annrheumdis-2019-eular.4089

\section{$\mathrm{AB} 0230$ CYTOKINE SIGNATURE DOES NOT CORRELATE WITH PAIN OR DISEASE ACTIVITY IN WELL CONTROLLED ERA}

Anna Radziszewska ${ }^{1}$, Corinne Fisher ${ }^{1}$, Timothy Bray ${ }^{2}$, Vera Choida ${ }^{2}$, Debajit Sen ${ }^{1}$, Margaret Hall-Craggs ${ }^{2}$, Coziana Ciurtin ${ }^{1} .{ }^{1}$ University College London, Arthritis Research UK Centre for Adolescent Rheumatology at UCLH, UCL, London, United Kingdom; ${ }^{2}$ University College London, Centre for Medical Imaging, London, United Kingdom

Background: Inflammation is a mediator and primary driver of joint damage in juvenile idiopathic arthritis (JIA). Musculoskeletal pain can be experienced in the presence or absence of inflammation. Enthesitis related arthritis (ERA) is a subtype of JIA characterised by inflammation of the spine, enthesis, and peripheral joints. Cytokines and numerous other regulatory molecules are implicated in pain and inflammation, yet, to date, no reliable biomarkers have been identified.

Objectives: To assess if cytokine profiles correlate with pain or disease activity in patients with ERA.

Methods: 42 patients, with either a prior diagnosis of JIA or back pain agreed to have a standard clinical MRI scan of lumbar spine, sacroiliac joints, and pelvis and to donate serum. Serum was also collected from 12 volunteer age matched healthy controls. Serum was analysed using a bead-based multiplex assay (Luminex) for the concentrations of the 
following analytes: IL-6, IL-12, IL-17, IL-23, IL-27, TNF $\alpha$, IFN $\gamma, \mathrm{MIF}, \mathrm{OPG}$, SOST, GM-CSF, VEGF, DKK-1, S100A8, MMP-3 and CRP. To assess pain levels, patients indicated on a scale of $0-10$ the amount of back pain experienced at night and separately the amount of back pain experienced at any time during the last week. They also completed a Bath Ankylosing Spondylitis Disease activity Index (BASDAI) questionnaire to assess disease activity. Cytokine concentrations in patients with ERA were compared with those of controls. The levels were also correlated with the two measures of back pain as well as individual questions from the BASDAI questionnaire.

Results: Of all 54 samples tested, 11 patients and 5 controls crossreacted with the negative control for the assay and thus were excluded from analysis. Based on MRI scan results, 14 patients had ERA, 8 had biomechanical pain, 7 had other subtypes of JIA, and 2 had non-specific features of spinal inflammation. The median overall back pain and the total BASDAI scores for the ERA group were 2.4 (IQR=1.45-5.55) and $2.5(\mathrm{IQR}=0.75-6.25)$ respectively, suggesting well controlled disease on treatment and minimal residual symptoms. There was no statistical difference between cytokine levels in the ERA group compared to controls when corrected for multiple testing, with the exception of IL-12 which was significantly higher in controls $(p=0.003)$. No correlation was found between cytokines and pain scores (at night or overall during the last week) or with the overall BASDAI score or any of the sub-component questions of the BASDAI questionnaire.

Conclusion: Well-controlled ERA patients on treatment have similar cytokine profiles as healthy controls and they do not correlate with clinical pain scores or disease activity.

Disclosure of Interests: None declared

DOI: 10.1136/annrheumdis-2019-eular.2827

\section{AB0230B STUDY OF CELIAC DISEASE ANTIBODIES IN PATIENTS WITH JUVENILE ONSET RHEUMATOLOGICAL DISORDERS}

Eman Hassan ${ }^{1,2}$, Nermen Magdy ${ }^{3,4} .{ }^{1}$ Egypt, Internal Medicine Rheumatology, Alexandria, Egypt, ${ }^{2}$ Faculty of Medicine, University of Alexandria, Internal Medicine Rheumatology, Alexandria, Egypt, ${ }^{1}$ Egypt, Internal Medicine Rheumatology, Alexandria, Egypt, ${ }^{4}$ Faculty of Medicine, Internal Medicine Rheumatology, Alexandria, Egypt

Background: Preclinical autoimmunity can be detected in the form of circulating autoantibodies in the peripheral blood many years preceding the onset of the clinical disease. Celiac disease (CD) is an immunological response to gluten in genetically susceptible people. The clinical presentation of CD includes abdominal pain, diarrhea and nutritional deficiencies. However; clinical symptoms could be misleading in most of the patients presenting subclinical forms with only minor gastroenterological symptoms. Celiac disease will present in older children by atypical presentation. Most celiac disease patients show atypical symptoms and may remain undiagnosed; which makes screening is mandatory in high-risk patients with autoimmune diseases. This collectively highlights the need to check for clinical evidence of celiac disease among patients diagnosed as juvenile onset rheumatic diseases

Objectives: The aim of the work is to screen for celiac disease antibodies (anti-tissue transglutaminase $\lg A$ and $\lg G$ auto-antibodies) in the serum of juvenile onset rheumatic diseases patients in comparison with normal subjects

Methods: Serum tTG (both $\lg A$ and $\lg$ ) level was detected in 60 juvenile onset rheumatic patients and 20 age and sex matched healthy controls. We also assessed different clinical and laboratory markers of disease parameters; 31 juvenile onset systemic lupus erythematosus (SLE), 21 juvenile onset idiopathic arthritis (JIA), 4 juvenile onset systemic sclerosis and 2 juvenile onset behcet's disease and each disease activity score. We also correlated serum transglutaminase auto-antibodies with each disease activity. Endoscopic examination and histopathologic examination, for the patients with a least one positive anti-tTG Ab was done

Results: Serum anti-tTG Abs was no statistical significant differences in the patients group than in the control group, and there was no correlation between anti-tTG Abs with positive disease activity score

Conclusion: The presence of concomitant $C D$ and another rheumatic disorder in the same patient is unlikely

\section{REFERENCES:}

[1] Roth B. Transglutaminase and peptidylarginine deiminase in the pathogenesis of autoimmune diseases. Doctorial Dissertation series. Sweden, Lund: Media-Tryck 2008
[2] Vojdani A. Antibodies as predictors of complex autoimmune diseases and cancer. International Journal of Immunopathology and Pharmacology. 2008; 21(3):553-566.

Disclosure of Interests: None declared

DOI: 10.1136/annrheumdis-2019-eular.848

\section{Rheumatoid arthritis - prognosis, predictors and outcome}

\section{AB0231 PAIN THRESHOLD IN RHEUMATOID ARTHRITIS AND ITS RELATED FACTOR}

Aysegul Altun Guvenir, Aslı Calıskan Uckun, Fatmagul Yurdakul, Hatice Bodur. Ankara N Hatice Bodur umune Training and Research Hospital, Physical Medicine and Rehabilitation, ANKARA, Turkey

Background: Pain remains the most important challenge for rheumatoid arthritis (RA) patients. In order to cope with pain in RA, clinicians firstly need to understand the degree of patients'pain. When self reported measures such as visual analog scale were used, inconsistency between clinicians' and patients' ratings of pain was demonstrated (1). As a result inability to fully assess the pain will cause the inability to fully assess the impact of pain on disease severity. Measuring pressure pain threshold with pressure algometry may provide additional advantages for the evaluation of pain and disease severity when compared with self-reported measures.

Objectives: The aim of this study was to evaluate the pain threshold and to determine the variables associated with pain threshold in the patients with RA.

Methods: The current study included 100 RA patients and 80 age-sex matched controls with non-inflammatory chronic low back pain. Clinica parameters, functional status, disease activity, pain, fatigue, depression, anxiety, pain catastrophizing and laboratory activity of RA patients were recorded. The pressure pain thresholds on the dominant thumb nail bed, trapezius and wrist of the two groups were measured by the algometer. The pain thresholds of the paients with RA and controls were compared. Moreover, the relation between pain threholds and all evaluated parameters in RA patients was analysed.

Results: The mean age of RA patients was $55.93 \pm 10.81$; the mean age of the controls was

$54,50 \pm 9,47$. The pain thresholds of RA patients and controls were statistically similar in all areas. When the parameters found to be significantly correlated with pain threshold in RA patients were analyzed by regression analysis, depression was the only factor associated with low pain threshold in all areas (Table 1).

Conclusion: We found that depression was the only factor associated with low pain threshold in the patients with RA. The use of pressure algometry in the evaluation of chronic pain could offer an additional method to detect pain/depression overlap.

Table 1. The correlation between pain threshold values and demographic, clinical and laboratory parameters of RA patients

\begin{tabular}{lcccccc}
\hline & \multicolumn{2}{c}{ Nail PT } & \multicolumn{2}{c}{ Wrist PT } & \multicolumn{2}{c}{ Trapezius PT } \\
\hline & $\mathrm{p}$ & $\mathrm{r}$ & $\mathrm{p}$ & $\mathrm{r}$ & $\mathrm{p}$ & $\mathrm{r}$ \\
\hline Age & 0,551 & 0,060 & 0,705 & 0,038 & 0,806 & 0,025 \\
BMI (kg/m ${ }^{2}$ ) & 0,174 & $-0,137$ & 0,066 & $-0,185$ & $0,046^{*}$ & $-0,200$ \\
$\begin{array}{l}\text { Duration of disease } \\
\text { (year) }\end{array}$ & 0,247 & $-0,117$ & 0,144 & $-0,147$ & 0,364 & 0,092 \\
Morning stiffness & & & & & & \\
(minute) & 0,415 & $-0,082$ & 0,592 & $-0,054$ & 0,542 & $-0,062$ \\
Tender joint counts & & & & & & \\
Swollen joint counts & $0,046^{*}$ & $-0,200$ & 0,099 & $-0,166$ & 0,361 & $-0,092$ \\
CRP & 0,109 & $-0,161$ & 0,227 & $-0,122$ & 0,522 & $-0,065$ \\
ESR & 0,681 & $-0,042$ & 0,144 & 0,147 & 0,076 & 0,178 \\
VAS & 0,952 & $-0,006$ & 0,330 & 0,098 & 0,453 & 0,076 \\
PCS & 0,056 & $-0,192$ & 0,069 & $-0,182$ & 0,086 & $-0,173$ \\
Fatigue Intensity Scale & $0,013^{*}$ & $-0,248$ & $0,013^{*}$ & $-0,249$ & $0,034^{*}$ & $-0,212$ \\
HADS-Anxiety & 0,107 & $-0,162$ & 0,145 & $-0,147$ & 0,422 & $-0,081$ \\
HADS-Depression & 0,157 & $-0,143$ & 0,118 & $-0,157$ & 0,285 & $-0,108$ \\
HADS-Total & $0,010^{*}$ & $-0,256$ & $0,005^{*}$ & $-0,282$ & $0,003^{*}$ & $-0,293$ \\
HAQ & $0,026^{*}$ & $-0,223$ & $0,014^{*}$ & $-0,245$ & $0,024^{*}$ & $-0,225$ \\
DAS28-ESR & $<0,001^{*}$ & $-0,264$ & $0,000^{*}$ & $-0,287$ & $<0,001^{*}$ & $-0,315$ \\
DAS28-CRP & $0,013^{*}$ & $-0,248$ & 0,091 & $-0,170$ & 0,211 & $-0,126$ \\
\hline
\end{tabular}

PT: pain threshold, BMI: Body mass index, PCS: Pain Catastrophizing Scale, VAS: Visual Analog Scale, HAQ: The health assessment questionnaire ${ }^{*} p<0.05$ 